\title{
PRIMER REGISTRO PALEOFLORISTICO DE LA FORMACION LA COLINA (PALEOZOICO SUPERIOR), CUENCA PAGANZO, REPUBLICA ARGENTINA
}

\author{
CARLOS O. LIMARINO' \\ SILVIA N. CESARI
}

\section{RESUMEN}

Se presenta una nueva asociación paleoflorística gondwánica hallada en los estratos rojos de la Formación La Colina, la cual era considerada estéril desde el punto de vista paleobotánico. Las plan. tas fósiles están preservadas como improntas en limolitas rojas algo calcáreas del miembro inferior de la formación. La asociación paleoflorística presenta las siguientes especies: Cordaites cf. hislopi (Bunbury) Seward \& Leslie, Euryphyllum whittianum Feist., Gangamopteris spp., Glossopteris cf. wilsonii (Seward) Archangelsky, Archangelsky \& Cúneo, ?Rhabdotaenia sp. y Paracalamites australis Rigby. Son discutidas la edad y las vinculaciones de esta tafoflora con otras equivalentes. La presencia de la flora de Glossopteris en la Formación La Colina atestigua una indudable edad pérmica para la misma.

\begin{abstract}
This paper presents a new paleofloristic gondwanic association discovered in the red beds of La Colina Formation, which has been considered previously as paleobotanically sterile. The fossil plants are preserved as impressions in red siltstone of the lower member of the formation. The paleo. floristic association is made up by the following genera: Cordaites cf. hislopi (Bunbury) Seward \& Leslie, Euryphyllum whittianum Feist., Gangamopteris spp.. Glossopteris cf. wilsonii (Seward) Archangelsky, Archangelsky \& Củneo, ?Rhabdotaenia sp., Paracalamites australis Rigby. The age and the correlation of this association are discussed. The presence of the Glossopteris flora in La Colina Formation confirms a Permian age for this unit.
\end{abstract}

\section{INTRODUCCION}

Los estratos rojos de la Formación La Colina culminan una secuencia sedimentaria continental ampliamente distribuida, depositada durante el neopaleozoico en la denominada Cuenca Paganzo, noroeste de la República Argentina. Esta formación cubre en forma concordante y transgresiva a la Formación Lagares (Carbónico superior) integrando ambas unidades litoestratigráficas el Grupo Paganzo (AZCUY \& MORELLI, 1970; AZCUY et al. 1979); el contacto superior resulta discordante con las sedimentitas triásicas de la Formación Amaná-Talampaya.

Las características litológicas de la Formación La Colina han permitido dividir a la unidad en dos miembros (AZCUY et al., op. cit.), el inferior integrado por areniscas, pelitas y conglomerados, de color rojo ladrillo y el superior formado en su mayor parte por areniscas finas de similar coloración.

El presente trabajo se refiere al contenido paleobotánico de la unidad sedimentaria, el tema resulta de particular interés si se tiene en cuenta que sus capas eran consideradas estériles desde el punto de vista de su contenido paleoflorístico. El material fue recolectado durante el levantamiento de un perfil detallado de la formación, lo cual permite una precisa ubicación estratigráfica de las capas portadoras. Este nuevo yacimiento se encuentra ubicado en el Mogote de la Desabrida, paraje situado a unos $7 \mathrm{~km}$ al NNW de la localidad de Amaná, provincia de La Rioja, Argentina (Fig. 1).

Los ejemplares se encuentran depositados en la colección de Paleontología del Departamento de Ciencias Geológicas de la Faculdad de Ciencias Exactas y Naturales de la Universidad de Buenos Aires. 


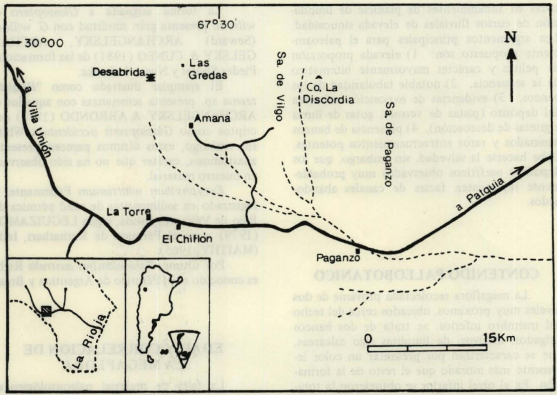

\section{LITOLOGIA DEL MIEMBRO INFERIOR}

Desde el punto de vista estratigráfico ambos miembros de la formación pueden ser reconocidos en el área; en particular interesa referirse al miembro inferior pues entre sus capas fue obtenida la megaflora que ocupa el presente trabajo. Se trata de una alternancia de areniscas (55\%) y pelitas (40\%), que se presentan en bancos medianos y gruesos (INGRAM. 1954), tabulares, de base plana, aunque en algunos casos las areniscas gruesas exhiben base levemente erosiva. El carácter bitemático de la secuencia es esporádicamente interrumpido por la aparición de cuerpos conglomerádicos, en general lenticulares, de escasa persistencia lateral y base erosiva; en estas capas es frecuen. te la presencia de abundantes individuos intra. formacionales que en algunos casos llegan a formar verdaderos niveles de conglomerados intraformacionales (heteroconglomerados en el sentido de SPALLETTI \& MAZZONI, 1975).

Las areniscas muestran una gran heterogeneidad granulométrica, aunque predomina las medianas y finas. Petrograficamente presentan una composición bastante constante; se trata de arenitas feldespáticas, de textura clástica con contactos tangenciales y en ocasiones flo- tantes; el cemento es por lo general calcáreo y menos frecuentemente ferruginoso. En lo referente a las estructuras sedimentarias, son frecuentes en el interior de las capas arenosas los entrecruzamientos de pequeña y mediana escala. En el trecho de los bancos, ondulitas asimétricas muy discontínuas pueden ser observadas.

Las pelitas se presentan por lo general en capas macizas y con menos frecuencia lamina. das; en el trecho de algunos estratos se observan pistas de vermes, gotas de lluvia y grietas de desecación de hasta $15 \mathrm{~cm}$ de diámetro. Ocasionalmente, las capas limosas contienen banquitos muy delgados de yeso y carbonato de calcio.

Con respecto a las psefitas, se trata de ortoconglomerados polimicticos medianos y finos, con clastos bien redondeados principal. mente de granito, feldespato y cuarzo; de formas preferentemente proladas y matriz arenosa guesa; los clastos frecuentemente se encuentran imbricados.

En lo que hace al paleoambiente sedimentario de estas rocas, LIMARINO (1983a, b) ha señalado la presencia de depósitos fluviales y eólicos en la Formación La Colina. En particular en el Mogote de la Desabrida el miembro inferior corresponde a sedimentitas. deposi. 
tadas en subambientes de planicie de inundación de cursos fluviales de elevada sinuosidad. Los argumentos principales para el paleoam. biente propuesto son: 1) elevada proporción de pelitas y carácter mayormente bitemático de la secuencia, 2) notable tabularidad de los bancos, 3) evidencias de exposición subaérea del depósito (pistas de vermes, gotas de lluvia y grietas de desecación), 4) presencia de bancos laminados y raros entrecruzamientos potentes. Debe hacerse la salvedad, sin embargo, que los depósitos psefíticos observados muy probablemente representen facies de canales abandonados.

\section{CONTENIDO.PALEOBOTANICO}

La megaflora recolectada proviene de dos niveles muy próximos, ubicados cerca del techo del miembro inferior, se trata de dos bancos delgados, masivos, de limolitas algo calcáreas. que se caracterizan por presentar un color levemente más morado que el resto de la forma. cion. En el nivel inferior se obtuvieron la totalidad de las impresiones de hojas mencionadas en este trabajo junto con algunos tallos flotados. En el nivel superior se hallaron numerosos moldes de equisetales, muchos en posición de crecimiento, junto con escasos restos indeterminables de hojas.

Debido al tipo de sedimento en que se han conservado, las improntas presentan un grado de preservación que dificulta su asignación específica. Además su coloración roja, sumada a la ausencia de una película carbonosa impiden la obtención de buenas fotografías, razón por la cual sólo son ilustrados algunos de los elementos de la asociación.

El análisis de la megaflora permite señalar la presencia de las siguientes formas (cuya descripción sistemática será objeto de una próxima contribución):

Cordaites $\mathrm{ef}$. hislopi (Bunbury) Seward \& Leslie Euryphyllum whittianum Feistmantel

Gangamopteris spp.

Glossopteris cf. wilsonii (Seward) Archangelsky, Archangelsky \& Cúneo.

?Rhabdotaenia sp.

Paracalamites australis Rigby

Los ejemplares determinados como Cor daites ef. hislopi (Bunbury) Seward \& Leslie, se asemejan a aquellos descriptos por ARCHANGELSKY \& ARRONDO (1973) como C. hislopi, y del mismo modo a material de las Sierras Australes de la provincia de Buenos Aires (HARRINGTON, 1933).
La forma asignada a Glossopteris cf. wilsonii presenta gran similitud con $G$. wilsonii (Seward) ARCHANGELSKY, ARCHANGELSKY \& CUNEO (1981) de las formaciones Piedra Shotel y Nueva Lubecka.

El ejemplar ilustrado como ?Rhabdotaenia sp. presenta semejanzas con aquellos de ARCHANGELSKY \& ARRONDO (1973) descriptos como Glossopteris occidentalis White, sin embargo, estos últimos parecen presentar anastomosis, caráter que no ha sido observado en nuestro material.

Euryphyllum whittianum Feistmantel es registrado en sedimentitas de edad pérmica del Bajo de Véliz, Argentina, según LEGUIZAMON (1979) y en el Pérmico de Karharbari, India (MAITHY, 1965).

Por último, Paracalamites australis Rigby es conocido en el Pérmico de Argentina y Brasil.

\section{EDAD Y CORRELACION DE LA MEGAFLORA}

La falta de material paleontológico en esta formación dió origen a opiniones diversas en cuanto a su edad en función que se utilizaran edades radimétricas, datos paleomagnéticos 0 correlación litológica con otras unidades consideradas equivalentes.

KEIDEL (1922) al ocuparse de estos estratos los consideró de edad triásica. Años más tarde, AZCUY \& MORELLI (1970) ubicaron estas rocas en el Pérmico según datos obtenidos por FRENGUELLI (1949) de niveles fosilíferos del Arroyo Totoral, los que fueron correlacionados con la Formación La Colina. BRA. CACCINI (1948) sobre la misma base había asignado al Carbónico superior o Pérmico inferior esta unidad.

Dataciones radimétricas de basaltos intercalados en la secuencia arrojaron una edad de $295 \pm 6$ m.a. (THOMPSON \& MITCHELL, 1972). AZCUY et al. (1979) en función de los resultados obtenidos por THOMPSON \& MITCHELL (op. cit.), VALENCIO (1972) y VA. LENCIO et al. (1977), estos dos últimos trabajos sobre datos paleomagnéticos, señalaron que la edad de la Formación abarca el EstefanianoKunguriano, acotando la misma al Carbónico superior-Pérmico inferior.

Los nuevos datos paleobotánicos presentados en este trabajo, indican una indudable edad pérmica para la Formación La Colina, especialmente si se tiene en cuenta la distribución temporal de las formas citadas, pues casi todas fueron anteriormente halladas. en aflo- 

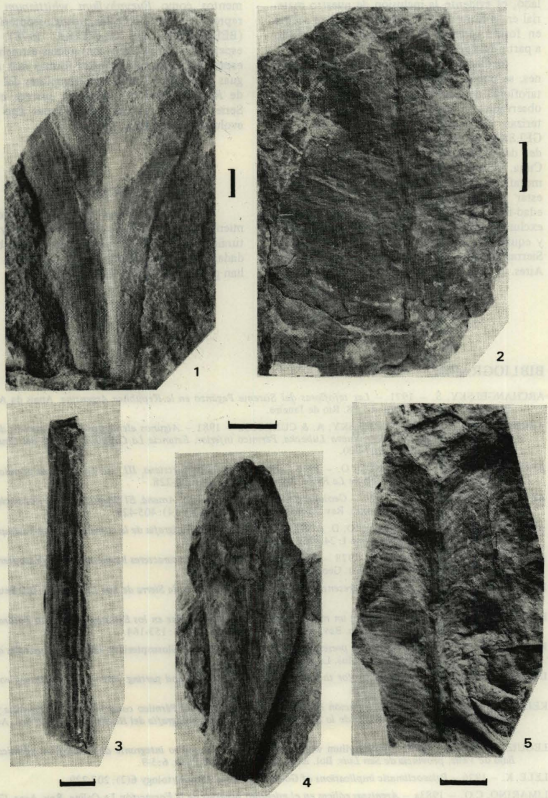

Fig. 1 - Euryphyllum whittianum Feistmantel; Fig. 2 - ?Rhabdotaenia sp.; Fig. 3 - Paracalamites australis Rigby; Fig. 4 - Gangamopteris sp.; Fig. 5-Glossopteris cf. wilsoni Anchangelsky. Archangelsky \& Cúneo. Escala gráfica $=1 \mathrm{~cm}$. 
ramientos pérmicos de Argentina. Por otro lado, es evidente la inclusión de nuestro material en la flora de Glossopteris, la que se acepta en forma generalizada, se habría desarrollado a partir del Pérmico.

Mediante el análisis de elementos comunes, se puede inferir un sincronismo con otras tafofloras de Argentina. Una gran similitud es observada con aquellas asociaciones que carac. terizan la edad-flora Lubeckense (ARCHANGELSKY, 1971), representada en las localidades de Bajo de Véliz, Arroyo de Totoral, Tasa Cuna, Nueva Lubecka. Sin embargo, en la megaflora de la Formación La Colina parecen estar ausentes numerosos integrantes de esa edad-flora, estando aparentemente constituida exclusivamente por glossopteridales, cordaitales y equisetales como es el caso del Pérmico de las Sierras Australes de la provincia de Buenos Aires.
Es interesante destacar la presencia de elementos como Euryphyllum whittianum que representa al grupo de las protoglossopterídeas (BERNARDES DE OLIVEIRA, 1978), una especie de Glossopteris con areólas estrechas y escasas que representaría las formas más antiguas de la flora, junto con ejemplares del tipo de Rhabdotaenia que en India aparece en la Serie Damuda representando formas algo más evolucionadas (LELE, 1976).

\section{AGRADECIMIENTOS}

Los autores desean expresar su agradecimiento a la Facultad de Ciencias Exactas y Naturales $y$ al CONICET por las facilidades brindadas. Del mismo modo, al Sr. Leonardo Killian por la confección de las fotografías.

\section{BIBLIOGRAFIA}

ARCHANGELSKY, S, - 1971 - Las tafofloras del Sistema Paganzo en la República Argentina. Anais da Acad. Bras. de Ciências, 43, Supl.: 67-88. Rio de Janeiro.

ARCHANGELSKY, S., ARCHANGELSKY, A. \& CUNEO, R. - 1981 - Algunos elementos paleofloristicos de las Formaciones Piedra Shotel y Nueva Lubecka, Pérmico inferior. Estancia La Casilda, provincia del Chubut. Ameghiniana XVIII (3-4):207-220.

ARCHANGELSKY, S. \& ARRONDO, O. - 1973 - Palqeophytologia Kurtziana, III. 10. La tafoflona permica de Sierra de Los Llanos, provincia de La Rioja. Ameghiniana 10 (3):220-228.

AZCUY, C. \& MORELLI, J. - 1970 - Geología de la comarca Paganzo - Amana. El Grupo Paganzo, Formaciones que lo componen y sus relaciones. Rev. Asoc. Geol. Argentina, XXV (4): 405-429.

AZCUY, C., MORELLI, J., VALENCIO, D. \& VILAS, J. - 1979 - Estratigrafía de la comarca A mand-Talampaya. Actas VII Congr. Geol Argentino I: 243-256. Neuquén.

BERNARDES DE OLIVEIRA, M. - 1978 - Ensaio sobre a utilizaçǟo de caracteres biométricos des glossopterialofitas em estratigrafia. Bol. IG. Inst. Geociencias, USP, 9:91-94.

BRACACCINI, 1. - 1948 - Sobre la presen cia del Carbonffero inferior en la Sierra de Los Llanos. Bol. Inf. Petrol.. 25:63-94.

FRENGUELLI, J, - 1949 - Acerca de un nuevo descubrimiento de plantas en los Estratos del Amoyo Totoral en la Sierra de los Llanos de La Rioja. Rev. Asoc. Geol. Argentina, IV (3): 153-164.

HARRINGTON, H. - 1933 - Sobre la presencia de restos de la Flora de Glossopteris en las Siemas Australes de la provincia de Buenos Aires. Rev. Mus. La Plata 34: 303-338.

INGRAM, R. - 1954 - Terminology for the thickness of stratification and parting units in sedimentary rocks. Geol. Soc, Am. Bull, 65:937-938.

KEIDEL, J. - 1922 - Sobre la distribución de los depositos giaciares del Pérmico conocidos en la Argentina y su significación para la estratigrafia de la Serie Gondwana y la paleogeografia del Hemisfério austral. Bol. Acad. Nac. Ciencias Córdoba, 25.

LEGUIZAMON, R, - 1979 - Euryphyllum whittianum Feistmantel, nuevo integrante de la tafoflora pérmica de Bajo de Veliz, provincia de San Luis. Bol. Asoc. Latin. Paleobot. Palin. 6:5-9.

LELE, K. - 1976 - Palaeoclimatic implications of Gondwana floras. Geophytology 6(2): 207-229.

LIMARINO, C.O. - 1983a - Areniscas eólicas en el miembro superior de la Formación La Colina. Rev. Asoc. Geol. Argentina. En prensa.

LIMARINO, C.O. - 1983b - Estudto de las rocas cldsticas y eruptivas vinculadas a la Cuenca Paganzo. Informe final CONICET. Inédito. 
MAITHY, R. - 1965 - Studies in the Glossopteris flora of India. Noeggerathiopsis and allied remains from the Karharbari beds. Girldh Coalfield, India, Palaeobotanist 13(1):94-100.

SPALLETTI, L. \& MAZZONI, M. - 1975 - Nuevo ondenamiento y significado de psefitas intraformacionales. Asoc. Arg. Min. Petr. Sed. 6(1-2): 1-10.

THOMPSON, R. \& MITCHELL, J. - 1972 - Paleomagnetic and radiometric evidence for the age of the lower boundary of the Kiaman magnetic interval in South America. Geophys. J.R. Astr. Soc. 27:207-214.

VALENCIO, D. - 1972 - Intercontinental correlation of the late Paleozoic South American rocks on the basis of their magnetic remanences. Anais Acad. Brasil. Cienc., 44, Supl.: 357-364.

VALENCIO, D., VILAS, J. \& MENDIA, J. - 1977 - Palaeomagnetism of a sequence of red beds of the middle and upper sections of Paganzo Group (Argen rina) and the correlation of Upper Paleozoic-Lower Mesozoic rocks. Geophys. J.R. Astr, Soc., 51:59-74. 